Pedagogía y Saberes $n .^{\circ} 52$

Universidad Pedagógica Nacional

Facultad de Educación. 2020. pp. 93-104

\title{
Tras las huellas de lo común. Significados de enseñanza en el Departamento de Pedagogía de la Universidad de Antioquia*
}

\section{Artículo de investigación}

\section{After the Traces of the Common. Meanings Attributed to Teaching in the Department of \\ Pedagogy of the University of Antioquia}

Seguindo as pegadas do comum. Significados atribuidos ao ensino no Departamento de

Pedagogia da Universidade de Antioquia

\section{Para citar este artículo:}

Parada M. y Hoyos E. (2020). Tras las huellas de lo común. Significados de enseñanza en el Departamento de Pedagogía de la Universidad de Antioquia. Pedagogía y Saberes, 52, 93-104. Dor: https://doi.org/10.17227/pys.num52-10018

* El presente trabajo es resultado de la investigación concluida, denominada "Análisis de los significados atribuidos a la enseñanza en la legislación de los programas de formación de maestros en Colombia, en los documentos analíticos y en algunos programas de los espacios de formación del Componente Común de formación inicial de maestros, de la Facultad de Educación de la Universidad de Antioquia", asesorada por los profesores Hilda Mar Rodríguez Gómez y Jesús Alberto Echeverri Sánchez. Agradecemos los indispensables aportes al texto por parte del profesor Marlon Espinosa.

** Profesor de la Universidad de Antioquia, estudiante del Doctorado en Sentidos, Teorías y Prácticas de la Educación de la Universidad Nacional del Litoral, (Argentina) e investigador del grupo Conversaciones entre Pedagogía y Psicoanálisis.

Correo electrónico: michael.parada@udea.edu.co Código oRCID: http://orcid.org/0000-0002-0778-3146

*** Profesor de la Universidad de Antioquia, magíster en Educación. Miembro del grupo de investigación Conversaciones entre Pedagogía y Psicoanálisis, e investigador del grupo Conversaciones entre Pedagogía y Psicoanálisis.

Correo electrónico: eliud.hoyos@udea.edu.co Código oRCID: http://orcid.org/0000-0003-3234-2913 


\title{
Resumen
}

En el presente artículo, resultado de investigación, se analizan diversas significaciones que adquiere la enseñanza en la formación contemporánea de maestros colombianos. Para ello se revisaron, a partir de un análisis documental riguroso, que tiene como base la lectura intratextual, documentos analíticos que forman parte del Componente Común de formación de maestros del Departamento de Pedagogía de la Facultad de Educación de la Universidad de Antioquia. El estudio permitió reconocer una amplia diversidad de significaciones, caracterizada por una proliferación desarticulada y difusa de las maneras de entender la enseñanza en el contexto de la formación de maestros. Con estas significaciones, arribamos a una discusión acerca de la enseñanza que pone en tensión una idea de lo común en la formación de maestros en dicha institución.

\section{Palabras clave}

pedagogía; formación de maestros; enseñanza; oficio

\begin{abstract}
The following article analyzes the diverse significances that teaching acquires in the contemporary education of Colombian teachers. For such purpose, this work carried out a rigorous documentary analysis on the analytical documents that belong to the 'Common Component of teachers' training from the Department of Pedagogy of the Faculty of Education of the University of Antioquia. The analysis allowed the recognition of a wide variety of significances, characterized by a diffuse and disjointed proliferation of ways of understanding teaching in the context of teacher training. Thus, we arrive at a discussion about teaching that puts an idea of what is common in teacher training in tension.
\end{abstract}

\section{Keywords}

pedagogy; teacher training; teaching; profession

\section{Resumo}

0 artigo a seguir analisa os diversos significados que o ensino adquire na formação contemporânea de professores colombianos. Para tanto, este trabalho realizou uma análise documental rigorosa sobre os documentos analíticos que fazem parte do 'Componente Comum de Formação de Professores' do Departamento de Pedagogia da Faculdade de Educação da Universidade de Antioquia. A análise permitiu o reconhecimento de uma ampla variedade de significados, caracterizada por uma proliferação difusa e desarticulada das formas de compreender o ensino no contexto da formação de professores. Assim, chegamos a uma discussão sobre o ensino que coloca uma idéia do que é comum na formação de professores em tensão.

\section{Palabras-chave}

pedagogia; formação de professores; ensino; profissão 


\section{Introducción}

$\mathrm{H}$ istóricamente, la Facultad de Educación de la Universidad de Antioquia ha tenido entre sus propósitos la formación de maestros. ${ }^{1}$ En el empeño de alcanzar dicho logro, ha puesto en circulación diversos tipos de saberes: los científicos específicos de cada programa; el cognitivo; el de la orientación y la consejería (dentro del cual, a su vez, se hicieron presentes pluralidad de tendencias); el didáctico y el pedagógico, entre otros.

De un tiempo para acá, ha habido un esfuerzo por consolidar un componente de formación común que encuentre en la pedagogía el fundamento para la formación de los licenciados. En un momento determinado, por ejemplo, se intentó asumir y concretar este propósito bajo el presupuesto de entender a la pedagogía como el saber fundante ${ }^{2}$ de la formación de maestros. Según Rafael Ríos (2006), la concepción de la pedagogía como saber fundante de los maestros fue un efecto político de los trabajos de investigación que hasta ese entonces había desarrollado el colectivo Historia de la Práctica Pedagógica, uno de los grupos de investigación que ha tenido influencia en la Facultad de Educación de la Universidad de Antioquia.

En la extensa obra del Grupo Historia de la Práctica Pedagógica (GHPP) — que ha logrado consolidarse como una tradición preponderante en la facultad y en el país- se puede apreciar que, a pesar de que ha sido producida en distintos momentos y que responde a diversos intereses, una vertiente de reflexión sobre la enseñanza ha estado presente, incluso, hasta nuestros días. Sobre todo, teniendo en cuenta uno de los planteamientos básicos sobre el cual se ha estructurado dicha reflexión: para la profesora Olga Lucía Zuluaga Garcés existe una "permanencia de la enseñanza como problema del saber pedagógico" (1999, p. 45). Esta no puede ser entendida, según la autora, como invariabilidad y ello permite afirmar, justamente, que la enseñanza es un problema constitutivo del pensamiento pedagógico, aun cuando su conceptualización varíe en el devenir del tiempo.

Así, en un contexto más contemporáneo, algunos miembros del GHPP y personas afines a él hallan que aquellos presupuestos iniciales acerca de la enseñanza admiten matices con el desarrollo, la preponderancia o presencia de otros discursos que alejan la reflexión sobre la enseñanza y enrarecen la

1 De aquí en adelante utilizaremos la expresión formación de maestros incluyendo también, obviamente, a las maestras.

2 Como se establece en los Decretos 3012 del 19 de diciembre de 1997 y 272 del 11 de febrero de 1998. Es preciso aclarar que ninguno de los decretos tiene vigencia en la actualidad. pedagogía. Dicha tensión estriba en que algunos de estos autores consideran, entre otras cosas, que la enseñanza "pierde tempranamente su hegemonía frente al aprendizaje" (Echeverri, 2015, p. 156). Para la profesora Araceli de Tezanos, que ha aportado reflexiones a lo que se podría denominar el campo pedagógico colombiano, la inclusión de conceptos de otros campos de las ciencias sociales al campo pedagógico - particularmente de las que llama corrientes psicológicas principales, esto es: el conductismo y el psicoanálisis, en tanto su "descubrimiento y reconocimiento de la existencia de la infancia" (2007, p. 11) desvía la reflexión sobre la enseñanza y centra "la acción pedagógica en el alumno, en tanto sujeto que aprende" (p. 11). Esta incursión conceptual, susceptible de historizar, de corrientes psicológicas al campo pedagógico, exige "del maestro una apertura hacia el conocimiento de los procesos que se generan en el niño y a aceptar este conocimiento como un nuevo parámetro para su acción" (Martínez, 2010, p. 155), en oposición a la pedagogía, en su versión de disciplina que estudia los conocimientos referentes a la enseñanza. Esto trae, según De Tezanos, al menos dos consecuencias: primera, un "desplazamiento del eje de la discusión sobre la formación -en la escueladesde el maestro hacia el alumno, abandonando toda discusión e inhibiendo el progreso de la investigación y producción de conocimiento sobre la enseñanza" (2007, p. 11); pero también, segunda, generaría una confusión conceptual entre los maestros, confusión que enrarecería la producción y el debate acerca de la enseñanza (p. 21).

Por su parte, el profesor Andrés Klaus Runge ha establecido un debate acerca del lugar del concepto de enseñanza en la pedagogía. Para Runge, la enseñanza es una forma particular que ha tomado la práctica educativa - entre otras - como una "interacción entre enseñantes y aprendices en la que a estos últimos se les ayuda, mediante diferentes métodos, a acceder al conocimiento y a desarrollar ciertas disposiciones" (s.f., pp. 2-3). Esto para decir que la enseñanza no es el concepto central de la pedagogía, sino uno más de los que se ocupa.

En la formación de maestros de la Facultad de Educación de la Universidad de Antioquia pueden aparecer estos y otros significados acerca de la enseñanza, debido a la pluralidad de enfoques teóricos presentes en los espacios de formación que pertenecen al Componente Común de formación de maestros. Entendimos, pues, en la investigación, que establecer dichos significados resulta relevante toda vez que la enseñanza es, como lo muestran los autores, al menos uno de los conceptos centrales para pensar la formación de los maestros, en cuanto enseñantes; 
es importante, visibilizar los trabajos y significados que, acerca de la enseñanza, hacen presencia en el Componente Común de formación de maestros. En consecuencia, el trabajo de investigación del cual emerge este artículo buscó analizar de qué maneras se significa la enseñanza en la legislación de los programas de formación de maestros en Colombia, en los documentos analíticos y algunos programas de los espacios de formación del Componente Común de la Facultad de Educación de la Universidad de Antioquia. Si bien hacemos mención a otras dos fuentes de indagación, como lo son la legislación y los espacios de formación del componente común, los hallazgos aquí presentados únicamente estarán relacionados con lo encontrado en los documentos analíticos del Componente Común.

\section{Horizonte conceptual}

Así pues, en busca de explorar cuáles son, hoy, las formas de presencia concretas que logra la enseñanza en dichos documentos analíticos, nos inscribimos conceptualmente en unos trabajos recientes que apuntan a visibilizar la importancia que tiene una formación de maestros apuntalada en la enseñanza - tanto en su dimensión práctica, como teórica-.

En esta línea, encontramos que Andrea Alliaud (2017) se propone reconocer el lugar que tiene, en la formación de maestros noveles, lo que ella denomina los saberes de la experiencia de los maestros. Hasta ahora, estos trabajos le han permitido advertir a la autora, por ejemplo, que "los docentes actuales no pueden o les cuesta enseñar porque nadie les ha enseñado a enseñar" (p. 95). Alliaud ofrece como hipótesis la idea de que esto quizá se debe a que la formación de maestros aporta "conocimientos, destrezas, habilidades, prácticas, pero no saberes" -que ella denomina de la experiencia-de oficio- que "parecen ser los grandes ausentes" (p. 73) del ámbito de la formación pedagógica de los maestros. De este modo, aprender el oficio de enseñar requiere de formación; es necesario, entonces, estar formado para enseñar, pero "formado no de cualquier manera" (p. 88). Para Alliaud (2017), el oficio de enseñar tendría que formar parte de todo el proceso formativo de los maestros y debería convocar el "saber que se produce al enseñar" (p. 82). Así pues, ¿qué tipo de formación es posible para los maestros si se tiene como horizonte la enseñanza?

La preocupación que alcanza la pregunta por la enseñanza en la formación de maestros requiere, a juicio de Alliaud (2017), "crear las condiciones pertinentes” para enseñar a enseñar (p. 96). En ese lugar, encuentra, al menos, tres condiciones: la primera se cifra en la responsabilidad de los formadores de maestros; la segunda, en lo que pueden aportar los conocimientos pedagógicos como apoyo al oficio de enseñar; y, la tercera, en lo que denomina - junto con Estanislao Antelo- una pedagogía específica de la formación de maestros.

En el primer caso, Alliaud (2017) afirma que la responsabilidad de "transmitir el oficio a quienes se están formando" (p. 96) es de los formadores de maestros, porque la enseñanza del oficio de enseñar exige formas de transmisión específicas y "situaciones especialmente creadas para que quienes se están formando puedan apropiarse de aquello que se transmite" (p. 88).

En la segunda condición, Alliaud y Antelo (2009) refieren que saber enseñar implica ensayar, ejercitar, probar. Pero "no se trata de probar de la nada o porque sí” (p. 98). Para que el ensayo, el ejercicio y la prueba, esto es, la práctica, se dé, hay que abrirle a la enseñanza una disponibilidad de saber, "hay que otorgarle una apoyatura específica, sobre la que será posible sostenerse" (p. 98). Alliaud (2017) asocia esta apoyatura a unas orientaciones previas a afrontar situaciones de enseñanza, cimientos necesarios para saber y poder enseñar hoy, que son "una responsabilidad que la formación docente inicial no puede eludir" (p. 71). A la manera de ver de Alliaud, (2017) dichos cimientos necesarios - que bien podrían denominarse conocimientos pedagógicos - son una referencia, "pero siempre estarán sujetos a ser revisados, re-creados, en función de lo que no sabíamos que iba a ocurrir y efectivamente ocurre" (p. 73), razón por la cual es necesario que dichos conocimientos "estén puestos al servicio de situaciones de enseñanza que permitan interrogarlas, enriquecerlas, imaginarlas de otra manera" (p. 158).

Esta última preocupación es la que lleva a Alliaud y Antelo a pensar en una formación específica de maestros - tercera condición- que incluya particularmente a la enseñanza. Se trata de una formación de maestros en la que no se pretenda que la pedagogía ocupe el lugar de teoría totalizante que dé respuestas anticipadas a lo que va a acontecer. Antes bien, se trata de una formación que favorezca precisamente "procesos formativos y de acompañamiento que brinden los gajes del oficio, es decir, prendas o señales de que se está preparado para aceptar el reto de enseñar" (Alliaud y Antelo, 2009, p. 100), en los que la pedagogía sería, más bien, "un discurso integrador y abarcador en el que los abordajes políticos, históricos, filosóficos y culturales del campo educativo adquieran sentido en la medida en que den sentido 
[sic] a las prácticas de enseñar" (Alliaud, 2017, p. 96). Así mismo, Alliaud y Antelo (2009) consideran que es posible contribuir al diseño de una formación de maestros poniendo a "la enseñanza y al oficio en el centro de la reflexión” (p. 93). Aquí, se entiende la formación docente inicial no solo como una instancia pertinente para difundir los cambios educativos, sino como un ámbito "definido conforme a las necesidades emergentes de los nuevos procesos de trabajo y de las nuevas y complejas condiciones escolares en que la enseñanza se ejerce" (Alliaud y Antelo, 2009, p. 93).

Somos nosotros quienes resaltamos la idea de una formación docente definida conforme a la enseñanza. En este sentido, Flavia Terigi (2004) realiza un planteamiento que califica de provocador: asegura que se puede pensar la enseñanza como un problema político. Esto significa, al decir de la autora, que la enseñanza puede ser entendida como "un problema que requiere un nivel de reflexión y de propuesta en un plano en el que su aparición es inhabitual: el plano político" (p. 192), lo cual implica inaugurar una perspectiva en que la enseñanza "acceda al rango de problema institucional” (p. 192). Y aunque la autora inscribe lo institucional en el plano de las políticas públicas, también es posible, creemos, que una Facultad de Educación funja como plano institucional en el que se perfile la enseñanza como problema político. Esto se puede pensar así toda vez que, asegura, "en el nivel local de la práctica pedagógica se juega el proyecto político" (Terigi, 2004, p. 193). Por tanto, politizar la enseñanza significa instalarla en un nivel de discusión que alcance un plano político e institucional.

Por su parte, el desconocimiento de la enseñanza como problema político por parte de las políticas educativas públicas tiene, para Terigi, al menos tres consecuencias. En primer lugar, crea discursos y estrategias para una formación de maestros sin ligarla concretamente con el mejoramiento de la calidad de la enseñanza (Terigi, 2004). Por otra parte, empobrece la planeación en relación con el conocimiento fundado existente al respecto de las formas específicas en que la educación se convierte en experiencia para los sujetos. Pero "esa pobreza no resulta tanto de la ausencia de saber fundado, sino del persistente desconocimiento de ese saber por parte de muchos de quienes asumen la responsabilidad del diseño de las políticas" (Terigi, 2004, p. 195). Finalmente, tercera consecuencia -y derivada de la anterior-, es que algunos proyectos de política educativa tienen que asumir una responsabilidad "ineludible cuando la evaluamos en relación con la disponibilidad de saber" (p. 198).
Esto último nos permite decir que la indagación por el saber disponible acerca de la enseñanza en los documentos analíticos del Componente Común permitirá también explorar y establecer la (posible) responsabilidad institucional, en lo relativo a la enseñanza, de una instancia crucial en dicha formación: la Facultad de Educación de la Universidad de Antioquia. Lo anterior significa atender especialmente a lo que se habilita desde su Componente Común de formación de maestros para - posiblemente- pensar la enseñanza como problema político inscrito en un plano institucional particular.

Así pues, en la presente investigación se trató de explorar los significados que se le atribuyen a la enseñanza en el marco de la formación pedagógica de los maestros de la Facultad de Educación de la Universidad de Antioquia, puesto que saber y poder enseñar es un oficio que tiene un valor no solo individual sino que compete a todos: tanto a los maestros y a sus formadores, como también a las políticas públicas, a las instituciones educativas -dentro de las cuales incluimos a las de formación de maestros- y a sus integrantes. Para Alliaud (2017) lo más interesante de saber acerca de las concepciones, "representaciones e ideas que tengamos o intentemos promover acerca de la enseñanza" (p. 101), es reconocer que esto no se queda sin consecuencias en las decisiones formativas que se toman, las cuales afectan las clases que se imparten en el contexto de la formación de maestros. "De modo que, si la posibilidad de enseñar, obrar, intervenir se interrumpe o se degrada, los docentes se verán perjudicados, amenazados, no realizados, pero las consecuencias alcanzan a todos" (Alliaud, 2017, p. 105).

\section{Metodología}

Las fuentes de indagación elegidas para realizar el presente estudio fueron: 1) la legislación colombiana que rige los programas y las instituciones de formación de maestros en el país; 2) algunos programas de algunos espacios de formación del Componente Común de formación inicial de maestros de la Facultad de Educación de la Universidad de Antioquia; y 3) los documentos analíticos del Componente Común de formación inicial de maestros de dicha Facultad. Sin embargo, tal como lo mencionamos anteriormente, los hallazgos aquí presentados serán solamente aquellos relacionados con la última fuente de indagación. ${ }^{3}$

3 Los documentos analíticos fueron escritos en el año 2010 por profesores adscritos al Departamento de Pedagogía de la Universidad de Antioquia. Los documentos trabajados fueron: Documento analítico del espacio de formación Infancias y culturas 
El paradigma de esta investigación fue cualitativo, de tipo documental, fundamentado en la hermenéutica, la cual realiza una interpretación crítica de textos. Este paradigma sugiere una "producción de interpretaciones reconstruidas del mundo social" (Denzin y Lincoln, 2012, p. 29), con lo cual no se pretendió llegar a la verdad última de los textos analizados sino a unas interpretaciones que permitieran enriquecer la comprensión de las maneras en que se significa la enseñanza en los documentos analíticos del Componente Común de formación inicial de maestros de la Facultad de Educación de la Universidad de Antioquia.

Para este análisis riguroso de los textos se tuvo en cuenta una propuesta de temporalidad lógica hecha por Jacques Lacan (Pérez, 1997), la cual rige una indagación en tres momentos: el instante de ver, el tiempo para comprendery el momento para concluir. Ello significa que, si bien se pueden prefigurar unos tiempos metodológicos de modo lineal, ellos pueden entrecruzarse en cada tiempo de la investigación. En ese sentido, tuvimos un instante - confrontación general con el objeto- para identificar los significados que los documentos analíticos del Componente Común atribuyen a la enseñanza. Un tiempo - análisis y formulación de preguntas a los textos- donde analizamos esos textos, íbamos tras la huella de los autores, conceptos y teorías que fundamentan las maneras de significar la enseñanza. Y, por último, un momento - construcción de interpretaciones, síntesis-donde construimos conclusiones relativas a los alcances y límites de las formas en que es significada la enseñanza en el Componente Común de formación inicial de maestros de la Facultad de Educación de la Universidad de Antioquia.

Además de tener en cuenta los tres tiempos lógicos para trabajar los textos, nos servimos de los desarrollos del profesor Juan Fernando Pérez en su artículo "Elementos para una teoría de la lectura" (1997). Allí, él desarrolla de manera precisa tres tiempos en la interpretación de los textos. Para nuestro fin, también recurrimos a la lectura intratextual, la cual supone producir sentido solo a partir de los significantes que proveen los documentos analíticos objetos de esta investigación, puesto que nuestro

juveniles; Arte, estéticas y educación; Ciberculturas, medios y procesos educativos; Cognición, cultura y aprendizaje; Educación y sociedad: teorías y procesos; Historia, imágenes y concepciones de maestro; Pedagogía, inclusión y discapacidad; Políticas públicas y legislación educativa; Seminario Interdisciplinario PedagogíaSaberes, y Sujetos en el acto educativo. principio investigativo fue determinar qué se dice allí, antes que anteponerle, a los significados que estos documentos otorgan a la enseñanza, una norma ideal que la caracterizaría, o su definición última.

La lectura intratextual es, pues, un tiempo de lectura que no impulsa la restitución de la verdad de los textos, sino la exigencia de atender a lo que el otro dice. De ahí que este tipo de lectura le implica al lector, además de un conocimiento de los códigos lingüísticos que allí se utilizan (erudición exigible), una "suspensión de toda lectura o referencia adicional" (Pérez, 1997, p. 113), aun cuando se le presentare el caso en el que, para comprender adecuadamente su dimensión denotativa (es necesario que disponga de ella, mas no de todas sus connotaciones), tenga que recurrir a lo que la erudición le invoque a modo de requisito de lectura. Entonces, la intratextualidad supone eclipsar saberes previos, para así dejar que sea el texto mismo el que responda a las preguntas que se le formulan.

\section{Hallazgos}

A continuación presentaremos los hallazgos logrados a partir de la lectura de los documentos analíticos (Departamento de Pedagogía, 2010a, 2010b, 2010c, $2010 \mathrm{~d}, 2010 \mathrm{e}, 2010 \mathrm{f})$. No sobra decir que resguardaremos los nombres particulares de los espacios de formación, pues lo que interesó a esta investigación fue analizar cómo se significaba la enseñanza en la formación - pedagógica- de los maestros en los espacios de formación del Componente Común, antes que un señalamiento particular a cada uno de dichos espacios.

\section{Enseñanza y campo de la pedagogía}

Veremos en esta categoría la enseñanza como un concepto del campo de la pedagogía en general, y del campo conceptual y narrativo de la pedagogía, en particular. De otra parte, también, la enseñanza como un concepto de lo que el documento analítico denomina saber didáctico.

\section{La enseñanza como un concepto del campo de la pedagogía}

Para algunos de los documentos analíticos, la enseñanza es significada como uno de los conceptos de los que se ocupa la pedagogía. De esta manera, un documento analítico (Departamento de Pedagogía, 2010a) busca significar la enseñanza específicamente como concepto articulador de la pedagogía, en relación con 
las ciencias y los saberes; para esto, acoge la idea de diálogo de saberes. Así, este documento asegura que dicho diálogo es posible, en el caso concreto del vínculo de la pedagogía con la ciencia y los saberes, si se piensa "el cuerpo de interrogantes o la problemática pedagógica que permita ligar lo teórico, lo conceptual y lo práctico en relación con la enseñanza y su articulación a los saberes específicos" (Departamento de Pedagogía, 2010a, p. 3). De tal manera que el problema central de esta perspectiva es pensar "la articulación de la enseñanza con los saberes y tratar de precisar los problemas que implica dicha articulación como acontecimiento político discursivo" (p. 3).

Conviene subrayar, para la presente investigación, que, al parecer, en esta propuesta de diálogo de saberes, la enseñanza es el concepto de la pedagogía que vincula a esta última con la ciencia y los saberes. Llama la atención que la enseñanza sigue designada - y, tal vez, limitada, restringida, relegada - en su relación con lo que en unas ocasiones es nombrado como saberes específicos, en otras, problemas científicos, objetos de enseñanza, enseñanza de las disciplinas $\mathrm{u}$ objetos de conocimiento de las disciplinas. Es preciso resaltar, también, que a la relación de la enseñanza con los saberes y a los problemas que esa relación implica, se les otorgue el estatuto de acontecimiento político discursivo. Sería interesante que esto último alcanzara mayor desarrollo en el texto (Departamento de Pedagogía, 2010a).

A pesar de lo anterior, otro documento analítico asevera que la pedagogía no se restringe simplemente a enseñar conocimientos. Así, en la línea de pensar el acto educativo en su relación con el saber pedagógico, el documento asegura que, en una entrevista sobre Henry Giroux, Ramón Flecha afirma que la pedagogía no se reduce simplemente a enseñar conocimientos (Departamento de Pedagogía, 2010b, p. 14) sino que implica la formación de subjetividades humanas (p. 14), la producción de prácticas que ayudan a crear un mundo mejor y escuchar la voz de los otros. De este modo, resulta interesante que se invoque a la pedagogía para intentar precisar las posibles relaciones entre acto educativo y saber pedagógico. Aquí se ve cómo se plantea que enseñar conocimiento entra en tensión con la formación, la creación de un mundo mejor y la escucha de los otros. Lo anterior nos induce unas preguntas: ¿Se puede pensar una enseñanza encaminada a la formación? ¿Se puede enseñar sin desconocer las voces de los otros? ¿Se puede enseñar y escuchar a los otros? ¿Enseñar conocimientos es simple? Esta postura acerca del enseñar, ¿qué tradiciones pedagógicas (o didácticas) tiene en cuenta?
La enseñanza como un concepto del campo conceptual y narrativo de la pedagogía

Un documento analítico se suscribe particularmente a la propuesta de campo conceptual y narrativo de la pedagogía. Allí se dice que el seminario tiene por objetivo general conformar con los estudiantes un espacio para analizar y reflexionar las problemáticas que se presentan en "los diálogos del campo conceptual y narrativo de la pedagogía con las ciencias y saberes como condición de posibilidad para los procesos de construcción y funcionamiento de la didáctica" (Departamento de Pedagogía, 2010a, p.1). Además, declara la necesidad de configurar, desde el campo conceptual y narrativo de la pedagogía, "una mirada sobre la enseñanza de las ciencias y de los saberes [que] posibilite una posición para el sujeto de la pedagogía que le permita plantear nuevas formas de ver y de decir para el desarrollo de las prácticas de enseñanza" (p. 1).

Para esta investigación, llama la atención que la posición que se procura en el seminario no ha de ser sin fundamento. Quizá lo que parece plantearse es una posición fundamentada -en los diálogos del campo conceptual y narrativo de la pedagogía con las ciencias y saberes - con consecuencias en las formas de ver y decir del sujeto de la pedagogía acerca de las prácticas de enseñanza. Sin embargo, no hay elementos textuales que permitan establecer quién sería el sujeto de la pedagogía. Resulta oportuno esclarecer, además, si por entender la pedagogía como campo - conceptual y narrativo- sería preciso considerar a alguien como el sujeto de la pedagogía, o si la noción -o concepto- de campo admite atribuir su agenciamiento a un sujeto en particular. Esto último nos pone en la dirección de preguntarnos entonces por la relación entre la noción - o concepto- de campo conceptual y narrativo de la pedagogía con la enseñanza, en el sentido de pensar si el sujeto de la pedagogía sería el mismo sujeto de la enseñanza.

\section{La enseñanza como un concepto del saber didáctico}

En la orientación de entender la enseñanza como concepto del saber didáctico, un documento analítico, en el marco de pensar la irrupción de lo que el texto llama régimen de saber científico en el denominado régimen de saber pedagógico-didáctico (Departamento de Pedagogía, 2010a, p. 4), dice que dichas irrupciones condicionan el concepto científico cuando se lo pasa a la racionalidad constitutiva de la didáctica, lo que implica trabajar interrogantes propios de los 
diálogos entre saberes y sus reglas de juego. Así, la construcción del saber didáctico implica lo cultural, lo social y lo científico de la ciencia a enseñar, pero también de "otras ciencias y saberes que pueden entrar a colaborar en el proceso que permite hacer explícita la enseñanza" (Departamento de Pedagogía, 2010a, p. 4). Esta implicación exige renovación y actualización del saber didáctico - que junto a esas otras ciencias y saberes permite hacer explícita la enseñanza- para que no sea caduco.

Por otra parte, en correspondencia con una imagen de ciencia como "proceso siempre inacabado de sentidos y finalidades diversas" (p. 4), la didáctica es presentada en este mismo documento como algo que no se puede confundir con el método o los medios (p. 4) de enseñanza. El texto asegura que el saber que se enseña también exige un movimiento permanente para mantener una coherencia (p. 4) entre el sistema de enseñanza y su entorno.

Como se puede advertir, resalta el hecho de no dejar relegada la didáctica al método o a los medios y de concebir el saber didáctico como algo dinámico, por cuanto complejiza a la enseñanza al ponerla en relación con otras ciencias y saberes. Queda por pensar lo que podría significar la llamada coherencia entre el sistema de enseñanza y su entorno.

Lo anterior nos sugiere algunas preguntas: $¿ E l$ entorno se refiere al disciplinar, al institucional, al de la enseñanza, al del saber que se enseña? Y si es así, ¿con cuál de todos es necesario que el sistema de enseñanza esté en coherencia?, ¿con todos? ¿La exigida coherencia no entra en conflicto y contradicción con las tensiones propias de las irrupciones que se reconocen cuando del régimen de saber científico se pasa al régimen de saber pedagógico-didáctico?

\section{Relación (tensa) entre la enseñanza y el aprendizaje}

En algunos documentos analíticos se refiere a la enseñanza y el aprendizaje como una relación. Veremos, a continuación, tres maneras diversas de presentarla.

\section{Relación directa: proceso de enseñanza/aprendizaje}

En el marco de la pregunta por el concepto de cibercultura, entendida como campo de conocimiento (Departamento de Pedagogía, 2010c, p. 5), uno de los documentos analíticos plantea la necesidad de desarrollar las implicaciones que este campo tiene en lo pedagógico y educativo (p. 6). Sobre todo, cuando "predomina la tendencia en la que se han impuesto los discursos tecnológicos sobre los pedagógicos" (p. 6). La cibercultura como fenómeno tecno-sociocultural es, entonces, una estrategia que todavía no se integra "formalmente a los procesos de enseñanzaaprendizaje en el nivel universitario" (p. 6).

Reconstruir la idea de este modo permite, al menos, tres comentarios. Por un lado, la enseñanza aparece ligada explícitamente al aprendizaje. Por otro, al aunar los procesos de enseñanza/aprendizaje al ámbito universitario, parece sugerirse un descuido de la escuela y otros espacios educativos, que es uno de los lugares a los cuales los maestros de la facultad podrían dirigirse. Esto advierte que la formación en cibercultura por parte de los maestros puede restringirse al ámbito de su propio aprendizaje, sin que ello implique, necesariamente, una reflexión por su enseñanza. Y, por último, es posible reconocer que entender como un proceso directo la enseñanza y el aprendizaje es un debate que algunos trabajos pedagógicos permiten superar.

\section{Hay relación enseñanza y aprendizaje, pero asincrónica}

Un documento analítico refiere que la enseñanza tiene relación con el aprendizaje, pero de manera asincrónica. En el ámbito de pensar y proponer conexiones posibles entre el espacio de formación y otros del Componente Común, este documento analítico asevera que las posibilidades de nexo con uno de los cursos precisan de un "exigente trabajo de recontextualización que tiene como tarea central la lectura de saberes, prácticas, lenguajes y sujetos, todo lo cual se entreteje de manera compleja dando configuración al contexto en el que se instalan las prácticas educativas" (Departamento de Pedagogía, 2010d, p. 13). En ese trabajo de recontextualización puede tener presencia, entre otros aspectos, la relación que, aunque asincrónica, se presenta entre el aprendizaje, como concepto y como práctica, con la enseñanza, ambas agenciadas por sujetos inscritos en prácticas, saberes y lenguajes. Así pues, la "condición de posibilidad del enseñar depende de la existencia de un sujeto dispuesto para la construcción de una relación de saber y dispuesto para el aprender (p.13).

Para el presente trabajo estimamos pertinente destacar el reconocimiento del enseñar como algo distinto del aprender, aunque en relación. Es posible reconocer allí una distinción que puede tener un sustento teórico - que no se hace explícito- pues reconoce que existe una acción vinculante entre el enseñar y el aprender aunque constituyan una relación asincrónica. Esto nos advierte la imposibilidad de relacionar directa y proporcionalmente a la enseñanza con el aprendizaje; ambas se dan en 
un tiempo que no necesariamente coincide, pero ello no descarta una relación. A su vez, esto permite interrogar el renombrado proceso de enseñanza/ aprendizaje. Por otra parte, al menos en el apartado presentado del documento se toman como sinónimos el enseñar y la enseñanza. Aunque en un fragmento anterior se entiende esta última como formas de enseñar, el documento no precisa si se restringe a entender la enseñanza solo como las formas que adopta el enseñar.

\section{Relación enseñanza y aprendizaje como coincidencia de sus términos}

Para concluir este apartado, en la idea de establecer - a propósito de la enseñanza- las posibles diferencias que existen entre lo que denomina acto educativo y saber pedagógico, un documento analítico dice que el acto educativo "debe ser esencialmente pedagógico" (Departamento de Pedagogía, 2010b, p. 13). De este modo, el profesional de la pedagogía -que el documento equipara al docente- es aquel que "busca hacer coincidir en un acto la enseñanza y el aprendizaje" (p. 13). Citando a Marcel Postic, el documento dice también que Skinner define "la enseñanza como la organización del aprendizaje" (p. 13).

En lo concerniente al presente trabajo, conviene señalar que el documento analítico reconoce como acto educativo a la enseñanza, al tiempo que la vincula con la pedagogía. Equipara al docente con el pedagogo sin mayores precisiones al respecto. En este sentido, podemos preguntarnos: $¿$ Qué hace al docente un pedagogo?, ¿qué hace al pedagogo un docente? ¿Qué hace al pedagogo, pedagogo? Pero, además, funde en un mismo acto a la enseñanza y al aprendizaje, lo cual alienta otras preguntas: ¿Lo que se enseña coincide con lo que se aprende, en caso de que se aprenda? $¿$ El aprendizaje es inmediato a la enseñanza? Es interesante la definición que, se dice, tiene Skinner acerca de la enseñanza. Esta nos suscita algunas preguntas más: ¿Qué significa organizar el aprendizaje? ¿Es organizar un sinónimo de controlar? ¿La enseñanza puede organizar el aprendizaje? ¿Si la enseñanza organiza el aprendizaje, se puede hablar de una relación?, ¿en qué términos? ¿Es el aprendizaje la medida de la enseñanza?

\section{El maestro como enseñante}

En consonancia con las dimensiones constitutivas de la formación y la acción pedagógica formuladas en la propuesta de Componente Común, particularmente con la segunda dimensión, a saber: el maestro como enseñante de las ciencias, las artes y la tecnología, fueron varios los documentos analíticos (Departamento de Pedagogía, 2010e, 2010f, 2010a) que acogieron la hipótesis del maestro como enseñante, aunque con algunas variaciones, como lo veremos a continuación.

\section{El maestro es competente para enseñar un saber, pero hay que pensarlo más allá de la simple enseñanza de la disciplina}

El eje sobre el cual reposan las reflexiones de un espacio de formación es la pregunta por la configuración histórica del sujeto/maestro, de ahí que su documento analítico (Departamento de Pedagogía, 2010e) muestra la importancia de pensar al maestro en la actualidad. Siguiendo la idea de que la época contemporánea plantea unos desafíos a la pedagogía y al maestro hoy, el documento analítico afirma que el sistema educativo colombiano está inmerso en permanentes cambios que cuestionan, entre otras, las funciones del maestro y "sus competencias para enseñar un saber" (Departamento de Pedagogía, 2010e, p. 3). Esta "actualidad [...] una reflexión crítica sobre las diversas formas de existencia del maestro y sobre los procesos que orientan su formación" (p. $3)$. Es así como el documento se apoya en Alberto Martínez Boom para pensar al maestro en relación con las preguntas y problemáticas actuales, "desde lo múltiple, desde lo diverso, desde otros escenarios que permitan reflexionarlo en y más allá del salón de clase, más allá de la simple enseñanza de la disciplina" (p. 3).

Para el presente estudio conviene resaltar que el documento analítico establezca que son los permanentes cambios sociales, culturales y políticos $-\mathrm{y}$ no nuestro sistema educativo - los que interpelan al maestro en sus competencias - y no en su saberpara enseñar. Llama la atención entonces que el enseñar un saber sea producto de la adquisición de unas competencias y no el resultado de un proceso de formación que contenga problematizaciones acerca de qué significa enseñar, cuál ha sido la historia de dichas problematizaciones y qué lugar ha tenido el enseñar en los modos en que se ha configurado el oficio del maestro históricamente. Además, llama la atención que el enseñar queda ligado a enseñar un saber, con lo cual parece suscribirse la idea de que la reflexión acerca del enseñar es propia de unos saberes específicos. Al mismo tiempo, al subrayar el carácter actual de algunas preguntas y problemáticas, el documento parece rehuir la preocupación que sustentaría a este espacio de formación: la inquietud por la configuración histórica del sujeto/maestro. 
La enseñanza: el quehacer de la docencia y del maestro

Un documento analítico define a la enseñanza como el quehacer de la docencia. De este modo, asegura que la configuración de una nueva política pública educativa, en el contexto latinoamericano y colombiano, exige también la creación de unas nuevas políticas no solo en el ámbito de la educación en general, sino de la formación docente en particular. De ahí que "la responsabilidad de las instituciones y programas de formación de docentes se debe concentrar en una mirada crítica a dichas políticas" (Departamento de Pedagogía, 2010f, p. 13) para no perder "el rumbo de lo que debe caracterizar el quehacer mismo de la docencia; la enseñanza, asumida intelectualmente desde la pedagogía" (p. 13); y para no aceptar una tesis que sacrifica la formación de maestros como intelectuales de la pedagogía, cuando desde las reformas se entiende la profesionalización de docentes como la adquisición de "unas competencias comunicativas e informativas como único requisito para garantizar la enseñanza efectiva" (p. 13).

Es preciso señalar, para el presente estudio, que el documento significa a la enseñanza como el quehacer mismo de la docencia que se asume "intelectualmente desde la pedagogía" (p. 13). Además, señala como algo inaceptable que el requisito exigido para garantizar una enseñanza efectiva se represente en "unas competencias comunicativas e informativas". Con esto parece indicar que la enseñanza no se resuelve con la adquisición de dichas competencias. Es necesario algo más, u otra cosa. La solución a este problema sugiere dos caminos: el primero, posicionamientos críticos frente a las políticas por parte de los intelectuales de la pedagogía; y, segundo, la asunción de la enseñanza intelectualmente desde la pedagogía. Sin embargo, este segundo camino no logra ser esclarecido por el documento.

\section{La enseñanza: una práctica que puede ser problematizada por el maestro}

La enseñanza aparece significada como una práctica que puede ser problematizada y el maestro justamente como quien la problematiza. Uno de los documentos analíticos declara como una de las finalidades del espacio de formación la configuración de espacios interdisciplinarios, donde el diálogo de saberes entre enseñantes de las ciencias pueda problematizar las prácticas de enseñanza en la perspectiva de elaborar colectivamente propuestas didácticas que reconozcan "las tensiones que se establecen entre los interrogantes propios del campo conceptual y narrativo de la pedagogía y el campo de educación en ciencias"
(Departamento de Pedagogía, 2010a, p. 6). Es de esta manera como el documento plantea como objetivos del espacio de formación analizar y reflexionar las prácticas pedagógicas del maestro para reconocer las formas como se ha constituido y reconocido como sujeto. En este marco, el texto afirma que es necesario pensar en las prácticas de los maestros en formación, los efectos de saber y las formas de verdad que se imponen, porque "el saber del maestro queda marcado" (p. 5) por su formación. Así, la pregunta por lo que "configuraría la experiencia del maestro" (p. 6) es necesaria para que sea él quien "problematice la enseñanza y las prácticas de ella que hasta el momento ha repetido sin interrogarlas" (p. 6), de modo que pueda elaborar prácticas que le permitan un "nuevo modo de pensar y de actuar" (p. 6).

En lo que respecta a la presente investigación, conviene resaltar el reconocimiento que el documento analítico le otorga a la responsabilidad que tienen las instancias formadoras de maestros, toda vez que ellas marcan el saber que ellos obtienen y con el cual afrontan sus prácticas. La idea de maestro problematizador de la enseñanza encuentra fundamento en la formación que reciba, de ahí que la interrogación de prácticas repetitivas y la creación de nuevas formas de pensar y actuar, pasan por lo que la formación les ofrezca. Ello parece indicar que es deseable una formación de maestros que presente, reflexione, complejice, conceptualice acerca de la enseñanza, desde puntos de vista teóricos y prácticos.

\section{La dispersión acerca de la enseñanza}

Finalmente, en el trabajo de investigación llevado a cabo encontramos una serie de significados acerca de la enseñanza que se caracterizan por la dispersión y que ofrecen escasas perspectivas de articulación con las categorías enunciadas anteriormente. Así, a continuación presentamos una de esas ideas sueltas que encontramos en relación con la enseñanza en los documentos analíticos.

\section{La enseñanza como objeto de las políticas educativas}

En uno de los documentos analíticos (Departamento de Pedagogía, 2010f) se presenta la enseñanza como un asunto a discernir en la formación de maestros desde el punto de vista de las políticas educativas. De esta forma, en la justificación del espacio de formación, el documento analítico asegura que los programas de formación de maestros requieren un espacio de conceptualización y reflexión sistemática para pensar las tendencias y perspectivas de la política pública en educación, pues determinan "la 
construcción y consolidación del sistema educativo en el que el docente tendrá su espacio de actuación profesional" (Departamento de Pedagogía, 2010f, p. 6). En este contexto, el documento asegura que es imprescindible que hoy los maestros se asuman como intelectuales de la educación lo cual significa ser "críticos frente al devenir de las políticas y las normas que enmarcan y dirigen el sistema educativo y sus repercusiones en el ámbito de la enseñanza" (p. 6), entre otras.

Para este estudio resulta pertinente destacar la posición que sugiere el documento analítico al indicar que uno de los asuntos frente a los cuales los maestros han de posicionarse es la enseñanza, sin descuidar otros como la pedagogía, el currículo o la evaluación. La idea de que las políticas tienen repercusiones en la enseñanza no soslaya una necesaria formación de los maestros en dos terrenos: el de las políticas, pero también el de la enseñanza, los cuales implican una lectura analítica del maestro que le permita un posicionamiento crítico. Conviene agregar que este documento nos da a pensar que la enseñanza se significa también en relación con las políticas educativas, aunque no presenta mayores desarrollos al respecto.

\section{A modo de "conclusión". De los posibles comunes...}

Creemos que hemos logrado una revisión detallada de las formas de significar la enseñanza en la legislación y en el Componente Común de formación de maestros de la Facultad de Educación de la Universidad de Antioquia. Sin embargo, si se quiere obtener un panorama más completo de lo que circula en la Facultad en relación con la enseñanza, sería preciso indagar, a nuestro modo de ver, en dos contextos más: el de las denominadas didácticas específicas de cada licenciatura y el de las prácticas, bien sea en las prácticas pedagógicas o en las llamadas prácticas tempranas, según se presenten en cada licenciatura. Antes bien, en su origen, el Componente Común tuvo la osadía de haber querido elevarse a la dignidad de un gesto institucional para establecer lo común. ${ }^{4}$ Sin embargo, hasta ahora $-y$, al menos, respecto de la enseñanza - en la facultad no hemos estado a la altura de este gesto y no hemos hecho justicia

4 Lo común no es lo homogéneo, ni más de lo mismo, ni lo único, ni la repetición; es, más bien, un punto de partida, un principio, que vislumbra un panorama posible en el cual se renuncia "al totalitarismo de lo uno y al esquematismo del dos, y que contrapone a ello la compleja simultaneidad de lo polifónico, plural y múltiple" (Frigerio y Diker, 2012, p. 8). académica a la prolífica producción que en el país existe a propósito de la enseñanza. Podemos decir esto en la medida en que, como hemos visto, en los documentos analizados no se presentaron tejidos a la manera de voces que, conservando su independencia, se tuvieran mutuamente en cuenta, lo que le podría dar lugar a la polifonía.

Conviene aclarar, pues, que no aspirábamos ni podíamos aspirar con esta investigación a encontrar homogeneidad en las formas de significar la enseñanza, pero es que lo común no es lo homogéneo, como dijimos. Ahora bien, como vimos, los posicionamientos en relación con la enseñanza son radicalmente divergentes las más de las veces, e incluso, por momentos, francamente opuestos. Valga decir, además, que podemos advertir una extraña escisión entre pedagogía y didáctica en la Facultad, de ahí que el Componente Común sea el escenario en donde se forman los estudiantes de las distintas licenciaturas en materia de pedagogía. Una posible implicación de lo anterior es que tal vez la separación que pudimos advertir tenga como efecto dificultades de articulación entre los llamados espacios de formación pedagógica y los espacios de formación en el campo de las denominadas didácticas específicas. Lo cual, a su vez, podría debilitar la reflexión, fundamentación y complejización de la enseñanza, en sentido práctico y teórico. Podemos anotar, además, que para algunas tradiciones pedagógicas la pedagogía y la didáctica no son campos separados.

De otra parte, podemos decir que el llamado desplazamiento de la enseñanza por el aprendizaje al que apelan algunos de los autores citados en la "Introducción", no se debe solo a la presencia de corrientes psicológicas en el campo educativo. Se debe también a la proliferación - desarticulada- de posiciones que acerca de la enseñanza hace presencia en el ámbito pedagógico de la formación de maestros de la Facultad de Educación de la Universidad de Antioquia, sin que por ello se hayan discutido ni definido mínimos comunes que permitan una orientación para dicha formación en lo que respecta a la enseñanza, precisamente. De hecho, como propone uno de los documentos analíticos (Departamento de Pedagogía, 2010d), la incursión de la psicología cultural puede significar la complejización del aprendizaje y también de la enseñanza, lo cual exige, eso sí, unos encuadres en los que se hagan explícitas las formas de entender la enseñanza, el aprendizaje y su posible relación.

Por otro lado, es posible decir que la enseñanza puede ser pensada desde el inicio de la formación de maestros y no solo cuando ellos emprenden sus prácticas. Así, nuestras prácticas de formación de 
maestros podrían estar orientadas por el saber de que disponemos en Colombia acerca de la enseñanza, si y solo si, instalamos y habilitamos dicho saber en los espacios de formación pedagógica de los estudiantes de las facultades de Educación. En este sentido, podemos afirmar que pensar la enseñanza como problema político implica considerarlo, a su vez, como un problema institucional de las facultades de Educación, que requiere y exige lineamientos curriculares institucionales que definan orientaciones y mínimos comunes para establecer lazos entre las diversas posturas y tradiciones para, con ello, no auspiciar la desarticulación que pudimos observar. Ahora bien, si se descuida la presencia de reflexiones fundadas acerca de la enseñanza en el plan de formación, se debilita también el campo de acción de los egresados de las facultades de Educación.

Hacia el final de esta investigación pudimos advertir que la pregunta por la enseñanza no era más que la antesala a una pregunta más amplia, que puede alcanzar profundas y variadas implicaciones: se trataría, en últimas, de pensar las formas de significar, hoy, la pedagogía en el contexto de las instituciones de formación de maestros en el país. Con todo, esta investigación no puede más que dejar sugerida la posibilidad de esta pregunta para eventuales indagaciones futuras.

\section{Referencias}

Alliaud, A. (2017). Los artesanos de la enseñanza. Acerca de la formación de maestros con oficio. Buenos Aires: Paidós.

Alliaud, A. y Antelo, E. (2009). Los gajes del oficio. Enseñanza, pedagogía y formación. Buenos Aires: Aique Educación.

Denzin, N. y Lincoln, Y. (2012). Manual de investigación cualitativa II. Paradigmas y perspectivas en disputa. Barcelona: Gedisa.

Departamento de Pedagogía. (2010a). Documento analítico del espacio de formación. Seminario interdisciplinario pedagogía-saberes. Medellín: Facultad de Educación, Universidad de Antioquia.

Departamento de Pedagogía. (2010b). Documento analítico del espacio de formación. Sujetos en el acto educativo. Medellín: Facultad de Educación, Universidad de Antioquia.
Departamento de Pedagogía. (2010c). Documento analítico del espacio de formación. Ciberculturas, medios y procesos educativos. Medellín: Facultad de Educación, Universidad de Antioquia.

Departamento de Pedagogía. (2010d). Documento analítico del espacio de formación. Cognición, cultura y aprendizaje. Medellín: Facultad de Educación, Universidad de Antioquia.

Departamento de Pedagogía. (2010e). Documento analítico del espacio de formación. Historia, imágenes y concepciones de maestro. Medellín: Facultad de Educación, Universidad de Antioquia.

Departamento de Pedagogía. (2010f). Documento analítico del espacio de formación. Políticas públicas y legislación educativa. Medellín: Facultad de Educación, Universidad de Antioquia.

De Tezanos, A. (2007). Oficio de enseñar-saber pedagógico: la relación fundante. Revista Educación y Ciudad, (12), 7-26.

Echeverri, J. (2015). Desplazamientos y efectos en la formación de un campo conceptual y narrativo de la pedagogía en Colombia (1989-2010). En J.A. Echeverri (ed.), Paradigmas y conceptos en educación y pedagogía (pp. 149-200). Bogotá: Siglo del Hombre.

Frigerio, G. y Diker, G. (2012). Educar: posiciones acerca de lo común. Paraná: Fundación La Hendija.

Martínez, A. (2010). ¿Puede la enseñanza incitar el pensamiento? En G. Frigerio. y G. Diker (comps.), Educar: ese acto político (pp. 153-171). Paraná: Del Estante.

Pérez, J. (1997). Elementos para una teoría de la lectura. Utopía Siglo XXI, 1(1), 111-126.

Ríos, R. (2006). De la pedagogía a las ciencias de la educación: una lectura desde el saber pedagógico colombiano. Revista Educación y Pedagogía, 18(44), 11-31.

Runge, A. (s.f.). ¿Educación o enseñanza?: El campo de las didácticas y las metódicas. Sin publicar. 1-19.

Terigi, F. (2004). La enseñanza como problema político. En G. Frigerio. y G. Diker (comps.), La transmisión en las sociedades, las instituciones y los sujetos. Un concepto de la educación en acción (pp. 191-202). Buenos Aires: Noveduc.

Zuluaga, O. (1999). Pedagogía e historia. La historicidad de la pedagogía. La enseñanza, un objeto de saber. Bogotá: Universidad de Antioquia, Anthropos, Siglo del Hombre. 